\title{
Approche morphozoométrique de chamelles (Camelus dromedarius L.) des populations algériennes Sahraoui et Targui
}

\author{
Baaissa Babelhadj ${ }^{1 *}$ Atika Benaissa ${ }^{2}$ Abdelkader Adamou ${ }^{2}$ \\ Faiza Tekkouk-Zemmouchi ${ }^{3}$ Soumia Raache ${ }^{2}$ \\ Taqiyeddine Babelhadj ${ }^{2}$ Claude Guintard ${ }^{4}$
}

\begin{abstract}
Mots-clés
Dromadaire, mensuration corporelle, population animale, Algérie
\end{abstract}

\footnotetext{
Submitted: 7 August 2017

Accepted: 30 October 2017

Published: 11 December 2017

DOI : $10.19182 /$ remvt.31483
}

\begin{abstract}
Résumé
L'objectif de cette étude était de comparer les caractérisations morphologiques et de déterminer l'indice de gracilité d'après des mesures zoométriques sur des chamelles adultes de deux populations algériennes, la Sahraoui et la Targui. Les mesures ont été réalisées sur des dromadaires femelles - 30 Sahraoui et 30 Targui - de plus de cinq ans dans le Sahara septentrional (région de Ouargla). Quatre mensurations ont été faites, puis l'indice de gracilité et le poids vif ont été calculés. Des équations de régression linéaire ont été proposées afin d'estimer le poids vif des animaux et la hauteur au garrot à partir des mensurations corrélées. Les valeurs moyennes des poids vifs des Sahraoui et des Targui étaient respectivement de $399 \pm 74 \mathrm{~kg}$ et de $428 \pm 82 \mathrm{~kg}$, pour une hauteur au garrot de 1,75 $\pm 0,08 \mathrm{~m}$ et de 1,81 $\pm 0,08 \mathrm{~m}$. Elles étaient respectivement de 0,96 $\pm 0,05 \mathrm{~m}$ et $0,97 \pm 0,08 \mathrm{~m}$ pour le vide sous-sternal, de 0,80 $\pm 0,08$ et 0,84 $\pm 0,07$ pour la profondeur thoracique, et de 1,20 $\pm 0,13 \mathrm{~m}$ et 1,18 $\pm 0,16 \mathrm{~m}$ pour l'indice de gracilité sous-sternal. Cette étude montre que la chamelle Targui est un peu plus gracile que la chamelle Sahraoui. Ces populations sont également associées à un ensemble de pratiques alimentaires, elles-mêmes liées à la qualité des parcours dont l'influence sur le développement morphologique des animaux a été largement observée.
\end{abstract}

- Pour citer cet article : Babelhadj B., Benaissa A., Adamou A., Tekkouk-Zemmouchi F., Raache S. Babelhadj T., Guintard C., 2017. Morphozoometric approach of female camels (Camelus dromedarius L.) of the Sahraoui and Targui Algerian populations. Rev. Elev. Med. Vet. Pays Trop., 70 (2): 65-69, doi: $10.19182 /$ remvt.31483

\section{INTRODUCTION}

Face aux changements climatiques, le dromadaire semble pouvoir s'adapter non seulement aux contraintes météorologiques

\footnotetext{
1. Ecole normale supérieure - Ourgla, Laboratoire de protection des écosystèmes en

1. Ecole normale supérieure - Ourgla, Laboratoire de protection des écosystèmes en
zones arides et semi-arides, Université Kasdi Merbah Ouargla, 30000 Ouargla, Algérie.

2. Laboratoire de protection des écosystèmes en zones arides et semi-arides, Université Kasdi Merbah Ouargla, 30000 Ouargla, Algérie.

3. Université Constantine 1, Institut des sciences vétérinaires, Laboratoire de gestion de la santé et des productions animales, El Khroub, Algérie.

4. Unité d'anatomie comparée, Ecole nationale vétérinaire de l'alimentation et de l'agroalimentaire, Nantes, France.

* Auteur pour la correspondance

Tél. : +213770582636; email : babelhadjbaaissa@gmail.com
}

(aridification du milieu), mais aussi à l'évolution des différents systèmes d'élevage et il représente ainsi un modèle animal très intéressant (Faye et al., 2012). L'activité cameline a de tout temps permis à une grande partie de la population pastorale d'en obtenir un revenu. Le nom des populations attribué à des groupes d'animaux varie en fonction des pays et des ethnies qui vivent dans ces zones (Abdallah et Faye, 2012 ; Chniter et al., 2013).

En ce qui concerne les dromadaires, la brévipédie (Saharaoui) et la longipédie (Targui) sont décrites depuis très longtemps (GeoffroySaint-Hilaire, 1861) et doivent être prises en compte (Babelhadj et al., 2016) dans le cadre d'une étude morphométrique. L'indice de gracilité sous-sternal est calculé selon la formule : IGS = VSS (vide soussternal)/PT (profondeur du thorax) (Bourzat et al., 1993). Ce travail vise à caractériser et à comparer la morphologie des deux populations, et à déterminer l'indice de gracilité des chamelles Sahraoui et Targui, qui sont les populations de dromadaires les plus rencontrées dans la région de Ouargla (Algérie). 


\section{MATERIEL ET METHODES}

\section{Les animaux}

L'étude a été réalisée sur 60 chamelles : 30 appartenaient à la population Sahraoui, dont le berceau est le Sahara septentrional (variété de la race Maghreb), et 30 appartenaient à la population Targui, dont le berceau se situe dans la région de Tamanrasset où se trouve la majorité des dromadaires de cette population. Ces deux populations appartenaient à des élevages semi-extensifs. Il s'agissait d'animaux âgés de plus de cinq ans utilisant des parcours distincts. Les deux types de dromadaires sont les plus répandus, les plus représentés et les mieux commercialisés dans la zone d'étude. Leur viande est largement consommée dans la région de Ouargla. L'échantillonnage a été sélectionné à l'abattoir de Ouargla de manière aléatoire, sans tenir compte de l'état de gravidité des chamelles.

La population Sahraoui représente un excellent animal de travail, pour la production de viande et de poils. Certaines femelles sont de très bonnes laitières. Son aire de répartition s'étend du grand Erg occidental au centre du Sahara (Benaissa, 1989) (figure 1).

Selon Messaoudi (1999), le dromadaire de la population Targui est un animal de course par excellence. Il est très haut sur des membres fins et secs, avec une robe grise à poils très courts et fins. C'est le dromadaire des Touaregs du Nord. Ce dromadaire est localisé au Sahara central, au Hoggar et à l'extrême Sud algérien (Tamanrasset). On peut le rencontrer un peu plus au nord, parce qu'il est très souvent utilisé comme reproducteur et comme animal de course. Il est utilisé aussi pour la selle et le bât (figure 2).

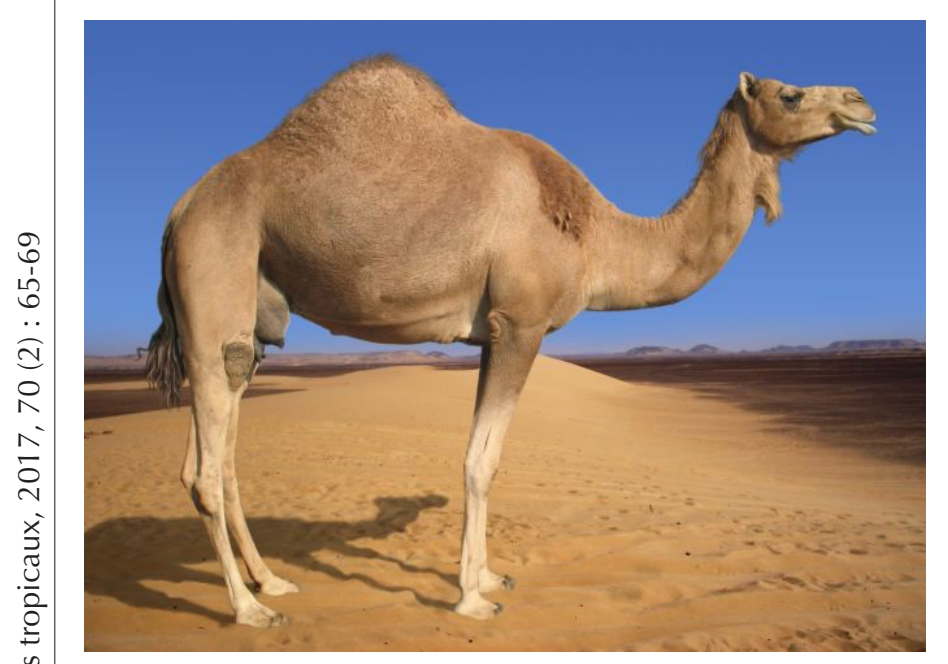

Figure 1 : dromadaire Sahraoui, Algérie.

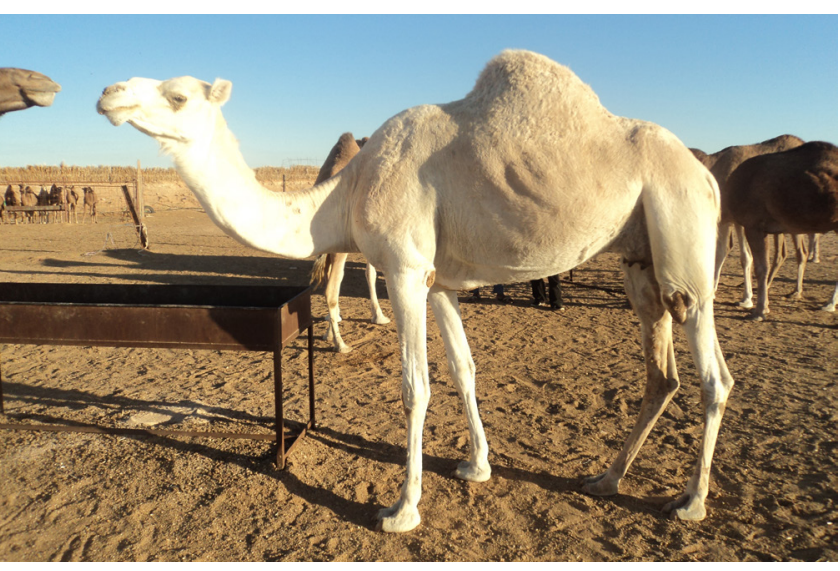

Figure 2 : dromadaire Targui, Algérie.

\section{La méthode}

Les mesures des différentes parties des individus vivants ont été réalisées en position debout (circonférences thoracique et abdominale) avec un ruban métrique. La hauteur au garrot (HG) et la profondeur de thorax (PT) ont été mesurées sous toise sur les 60 animaux.

Pour la détermination de l'âge, nous avons eu recours à l'expérience des éleveurs grâce à l'examen de la denture qui reste la méthode la plus couramment utilisée par les chameliers. La traduction de la chronologie dentaire chez le dromadaire conduit à des appellations locales telles que jedaa (5 ans), theni (6 ans), rebaa (7 ans), sdass (8 ans) et garah (9 ans). A partir de 10 ans, l'âge est estimé par le degré d'usure des dents.

Pour chaque individu, quatre mesures zoométriques ont été réalisées : la hauteur au garrot, le vide sous-sternal (VSS), la circonférence thoracique (CT) et la circonférence abdominale (CA). Les trois mensurations ont permis d'estimer le poids vif (PV) de l'animal, à partir de la formule barymétrique de Boué (1949) : PV $=53$ x CT x CA x HG. Cette formule a été choisie car elle se rapproche beaucoup de la plupart des formules proposées (Benaissa, 1989) et elle a déjà été appliquée sur les dromadaires du Maghreb ; elle rend ainsi les comparaisons possibles (Babelhadj et al., 2016).

Les données ont été saisies dans Microsoft Excel 2007. Les analyses des moyennes (test $t$ de Student) ont été réalisées avec le logiciel XL Stat (Addinsoft), au seuil de significativité de $p=0,05$.

\section{RESULTATS ET DISCUSSION}

Les tableaux I et II montrent les paramètres biométriques des chamelles Sahraoui et Targui âgées plus de cinq ans. Respectivement elles pesaient $399 \pm 74 \mathrm{~kg}$ et $428 \pm 82 \mathrm{~kg}$ pour une hauteur au garrot de $1,75 \pm 0,08 \mathrm{~m}$ et $1,81 \pm 0,08 \mathrm{~m}$. Les moyennes de vide sous-sternal chez les deux populations étaient presque égales. Les circonférences abdominales étaient très légèrement plus grandes chez la Targui que chez la Sahraoui mais sans différences significatives $(p=0,606)$. Nos résultats ne concordent pas avec ceux de Oulad Belkhir et al. (2013) pour la circonférence abdominale : pour la Saharaoui 1,638 $\pm 0,199$ vs 2,27 $\pm 0,22 \mathrm{~m}$ dans notre étude, et pour la Targui 2,200 $\pm 0,258$ vs $2,30 \pm 0,21 \mathrm{~m}$ dans notre étude.

Au-delà des valeurs moyennes, la variabilité globale du coefficient de variation $(\mathrm{CV})$ a été plus importante pour les variables pondérales $(0,19 \%$ pour le poids vif des deux populations) que pour les mesures linéaires (de 0,04 à 0,10\%). Parmi ces dernières, la hauteur au garrot a été le paramètre linéaire le moins variable $(\mathrm{CV}=0,04 \%)$. En ce qui concerne l'indice de gracilité, le $\mathrm{CV}$ a été légèrement plus important, proche de celui du poids (respectivement $0,19 \%$ et $0,14 \%$ ). L'écarttype des mesures zoométriques linéaires a donc été plus faible que celui des mesures pondérales.

La différence entre les moyennes des deux populations n'était pas significative, à l'exception de la hauteur au garrot et de la circonférence thoracique. La moyenne des indices de gracilité n'a pas montré de différences significatives entre les deux populations (tableau II). Les animaux semblaient donc relativement homogènes en matière de morphologie. En revanche, leur état d'embonpoint et leur taille ont été plus variables, traduisant un écart important des valeurs pondérales.

Parmi les paramètres barymétriques, certains étaient particulièrement bien corrélés entre eux de façon logique. Les valeurs de la matrice de corrélation de la Sahraoui (tableau III) ont montré que la circonférence thoracique était moyennement corrélée avec la hauteur au garrot. Chez la Targui la circonférence thoracique était sous la moyenne de corrélation avec la hauteur au garrot. Des résultats différents des nôtres ont été signalés par Oulad Belkhir et al. (2013) 


\section{Tableau I}

Paramètres biométriques des dromadaires femelles Sahraoui en Algérie

\begin{tabular}{|c|c|c|c|c|c|c|c|}
\hline Paramètres statistiques & $\begin{array}{l}\text { HG } \\
(\mathbf{m})\end{array}$ & VSS & CT & $\mathrm{CA}$ & PT & IGS & $\begin{array}{l}\text { PV } \\
\text { (kg) }\end{array}$ \\
\hline $\mathrm{n}$ & 30 & 30 & 30 & 30 & 30 & 30 & 30 \\
\hline Moyenne & 1,75 & 0,96 & 1,88 & 2,27 & 0,80 & 1,20 & 399 \\
\hline Minimum & 1,62 & 0,84 & 1,65 & 1,85 & 0,08 & 0,13 & 267 \\
\hline Maximum & 1,88 & 1,07 & 2,4 & 2,82 & 1,02 & 1,45 & 614 \\
\hline$\sigma$ & 0,08 & 0,05 & 0,16 & 0,22 & 0,08 & 0,13 & 74 \\
\hline $\mathrm{CV}(\%)$ & 4 & 5 & 9 & 10 & 10 & 19 & 19 \\
\hline
\end{tabular}

HG : hauteur au garrot ; VSS : vide sous-sternal ; CT : circonférence thoracique ; CA : circonférence abdominale ; PT : profondeur thoracique ; IGS : indice de gracilité sous-sternal ; PV : poids vif ; $\sigma$ : écart-type ; CV : coefficient de variation

\section{Tableau II}

Paramètres biométriques des dromadaires femelles Targui en Algérie

\begin{tabular}{|c|c|c|c|c|c|c|c|}
\hline Paramètres statistiques & $\begin{array}{l}\text { HG } \\
(\mathrm{m})\end{array}$ & VSS & CT & $\mathrm{CA}$ & PT & IGS & $\begin{array}{l}\text { PV } \\
\text { (kg) }\end{array}$ \\
\hline $\mathrm{n}$ & 30 & 30 & 30 & 30 & 30 & 30 & 30 \\
\hline Moyenne & 1,81 & 0,97 & 1,93 & 2,30 & 0,84 & 1,18 & 428 \\
\hline Minimum & 1,62 & 0,65 & 1,50 & 1,90 & 0,68 & 0,59 & 271 \\
\hline Maximum & 1,95 & 1,10 & 2,50 & 2,80 & 1,11 & 1,62 & 679 \\
\hline$\sigma$ & 0,08 & 0,08 & 0,18 & 0,21 & 0,07 & 0,16 & 82 \\
\hline CV $(\%)$ & 4 & 9 & 9 & 9 & 9 & 14 & 19 \\
\hline
\end{tabular}

HG : hauteur au garrot ; VSS : vide sous-sternal ; CT : circonférence thoracique ; CA : circonférence abdominale ; PT : profondeur thoracique ; IGS : indice de gracilité sous-sternal ; PV : poids vif ; $\sigma$ : écart-type ; CV : coefficient de variation

\section{Tableau III}

Matrice des corrélations des paramètres biométriques des dromadaires femelles Sahraoui et Targui en Algérie

\begin{tabular}{|c|c|c|c|c|c|c|c|}
\hline Sahraoui & HG & VSS & CT & CA & PT & IGS & PV \\
\hline HG & 1 & 0,608 & 0,588 & 0,224 & 0,771 & $-0,327$ & 0,614 \\
\hline VSS & 0,608 & 1 & 0,382 & $-0,048$ & $-0,037$ & 0,549 & 0,291 \\
\hline $\mathrm{CT}$ & 0,588 & 0,382 & 1 & 0,574 & 0,434 & $-0,154$ & 0,900 \\
\hline $\mathrm{CA}$ & 0,224 & $-0,048$ & 0,574 & 1 & 0,320 & $-0,270$ & 0,837 \\
\hline PT & 0,771 & $-0,037$ & 0,434 & 0,320 & 1 & $-0,852$ & 0,539 \\
\hline IGS & $-0,327$ & 0,549 & $-0,154$ & $-0,270$ & $-0,852$ & 1 & $-0,283$ \\
\hline PV & 0,614 & 0,291 & 0,900 & 0,837 & 0,539 & $-0,283$ & 1 \\
\hline Targui & HG & VSS & CT & $\mathrm{CA}$ & PT & IGS & PV \\
\hline HG & 1 & 0,584 & 0,409 & 0,331 & 0,394 & 0,055 & 0,560 \\
\hline VSS & 0,584 & 1 & 0,107 & $-0,177$ & $-0,516$ & 0,827 & 0,105 \\
\hline $\mathrm{CT}$ & 0,409 & 0,107 & 1 & 0,624 & 0,310 & $-0,177$ & 0,899 \\
\hline $\mathrm{CA}$ & 0,331 & $-0,177$ & 0,624 & 1 & 0,550 & $-0,452$ & 0,870 \\
\hline PT & 0,394 & $-0,516$ & 0,310 & 0,550 & 1 & $-0,879$ & 0,472 \\
\hline IGS & 0,055 & 0,827 & $-0,177$ & $-0,452$ & $-0,879$ & 1 & $-0,266$ \\
\hline PV & 0,560 & 0,105 & 0,899 & 0,870 & 0,472 & $-0,266$ & 1 \\
\hline
\end{tabular}

En gras, valeurs significatives (hors diagonale) $(\mathrm{p}=0,050)$ 
chez la Sahraoui : la hauteur au garrot est très faiblement corrélée à la circonférence thoracique et sous la moyenne de corrélation avec la circonférence abdominale. Pour ce qui est de la Targui les circonférences thoracique et abdominale sont corrélées avec la hauteur au garrot (Oulad Belkhir et al., 2013).

Les coefficients de corrélations étaient moins élevés mais toujours significatifs (tableau III) chez les chamelles Targui que chez les chamelles Sahraoui, notamment entre la hauteur au garrot, la circonférence abdominale, la circonférence thoracique et la profondeur thoracique. La dispersion des valeurs de la population Sahraoui était très voisine de celle de la population Targui, bien que cette dernière soit légèrement plus grande et plus lourde (Babelhadj et al., 2016). Des résultats proches de ceux enregistrés dans la population Sahraoui ont été enregistrés par d'autres auteurs ; la chamelle Targui est une meilleure laitière mais elle s'engraisserait aussi plus rapidement (Benyoucef et Bouzegag, 2006). Ces populations sont également associées à un ensemble de pratiques alimentaires, elles-mêmes liées à la qualité des parcours dont l'influence sur le développement morphologique des animaux a été largement soulignée (Babelhadj et al., 2016).

\section{REFERENCES}

Abdallah H.R., Faye B., 2012. Phenotypic classification of Saudi Arabian camel (Camelus dromedarius) by their body measurements. Emir. J. Food Agric., 24 (3): 272-280

Almathen F., Charruau P., Mohandesan E., Mwacharo J.M., Orozcoter Wengel P., Pitt D., Abdussamadg A.M., et al., 2016. Ancient and modern DNA reveal dynamics of domestication and cross-continental dispersal of the dromedary. PNAS, 113 (24): 6707-6712, doi: 10.1073/ pnas. 1519508113

Babelhadj B., 2017. Ostéo-biométrie et structure osseuse des métapodes de dromadaire (Camelus dromedarius L., 1758) : étude comparée de deux populations, Sahraoui et Targui. Thèse Doct. Sciences agronomiques, Université Kasdi Merbah Ouargla, Algérie, 202 p.

Babelhadj B., Abdelkader A., Tekkouk-Zemmouchi F., Benaissa A., Guintard C., 2016. Etude biométrique de dromadaires de deux populations algériennes : la Sahraoui et la Targui (Camelus dromedarius L.). Livest. Res. Rural Dev., 28 (2).

Ben Aissa R., 1989. Le dromadaire en Algérie. In : séminaire sur la digestion, la nutrition et I'alimentation du dromadaire, sémin. méditerr. $\mathrm{n}^{\circ}$ 2, Saragosse, Espagne (éd. Tisserand J.-L.). Ciheam Options Méditerr. Sér. A : 19-28. http:// ressources.ciheam.org/om/pdf/ a02/Cl000422.pdf

Benyoucef M.T., Bouzegag B., 2006. Résultats d'étude de la qualité de la viande de deux races camelines (Targui et Sahraoui) à Ouargla et Tamanrasset (Algérie). Ann. Inst. Nat. Agron. El-Harrach, 27 (1-2) : 37-53

\section{CONCLUSION}

En apparence, les deux populations de chamelles se distinguaient sensiblement sur le plan du gabarit. La chamelle Targui est considérée comme plus gracile que la chamelle Sahraoui, mais il s'agit plus d'une impression résultant de sa plus grande hauteur au garrot et de ses membres plus fins. En effet, l'indice de gracilité des deux populations est très voisin et n'est pas significativement différent d'un point de vue statistique. La Targui est certes plus élancée (HG en moyenne de $1,81 \mathrm{~m}$ vs $1,75 \mathrm{~m}$ pour la Sahraoui) mais elle a aussi une cage thoracique plus développée (en moyenne de 1,93 m vs 1,88 m pour la Sahraoui), de sorte que les conformations globales sont sensiblement les mêmes. L'étude de la morphométrie osseuse à partir des métapodes (Babelhadj, 2017) donne des résultats concordants avec ces données biométriques. Il existe donc une harmonie de l'ensemble de l'animal, tant au niveau de l'extérieur que de son squelette. Si le phénotype est ainsi bien décrit (Almathen et al., 2016), il pourrait être intéressant de mieux caractériser d'un point de vue génétique ces populations qui demeurent assez proches et dont l'histoire commune n'est pas si éloignée.
Boué A., 1949. Essai de barymétrie chez le dromadaire Nord-africain. Rev. Elev. Med. Vet. Pays Trop., 3 (1) : 13-16, doi : 10.19182/ remvt.6857

Bourzat D., Souvenir Zafindrajaona P., Lauvergne J.J., Zeuh V., 1993. A morpho-biometric comparison between goats from Northern Cameroon and Chad. Rev. Elev. Med. Vet. Pays trop., 46 (4): 667-674, doi : 10.19182/remvt.9423

Chniter M., Hammadi M., Khorchani T., Krit R., Benwahada A., Ben Hamouda M., 2013. Classification of Maghrebi camels (Camelus dromedarius) according to their tribal affiliation and body traits in southern Tunisia. Emir. J. Food Agric., 25 (8): 625-634

Faye B., Chaibou M., Vias G., 2012. Integrated impact of climate change and socioeconomic development on the evolution of camel farming systems. Br. J. Environ. Clim. Change, 2 (3): 227-244, doi: 10.9734/ BJECC/2012/1548

Geoffroy-Saint-Hilaire I., 1861. Acclimatation et domestication des animaux utiles. La maison rustique, Paris, France, 534 p.

Messaoudi B., 1999. Point de situation sur l'élevage camelin en Algérie. In : Les premières journées sur la recherche cameline de Ouargla, 25-26 mai 1999. Université de Ouargla, Algérie, 13-14

Oulad Belkhir A., Chehma A., Faye B., 2013. Phenotypic variability of two principal Algerian camel's populations (Targui and Sahraoui). Emir. J. Food Agric., 25 (3): 231-237, doi: doi.org/10.9755/ejfa. v25i3.15457 


\section{Summary}

Babelhadj B., Benaissa A., Adamou A., Tekkouk-Zemmouchi F., Raache S., Babelhadj T., Guintard C. Morphozoometric approach of female camels (Camelus dromedarius L.) of the Algerian Sahraoui and Targui populations

The objective of this study was to compare morphological traits and determine the gracility index of two Algerian camel populations, the Sahraoui and the Targui, based on zoometric measurements. These measurements concerned a sample of adult female camels - 30 Sahrawi and 30 Targui - in Northern Sahara (Ouargla region). Four measurements were taken, then the gracility index and live weights were calculated. Linear regression equations have been proposed to estimate the live weight of the animals and the height at the withers from correlated measurements. The mean live weights of the Sahraoui and Targui were $399 \pm 74 \mathrm{~kg}$ and $428 \pm 82$ $\mathrm{kg}$, respectively, for a height at the withers of $1.75 \pm 0.08 \mathrm{~m}$ and $1.81 \pm 0.08 \mathrm{~m}$. The mean substernal cavities measured $0.96 \pm 0.05 \mathrm{~m}$ and $0.97 \pm 0.08 \mathrm{~m}$, the mean thoracic depths $0.80 \pm 0.08$ and $0.84 \pm 0.07$, and the mean substernal gracility indices were $1.20 \pm 0.13 \mathrm{~m}$ and $1.18 \pm 0.16 \mathrm{~m}$. This study shows that the Targui female camel is a little more gracile than the Sahraoui. These populations are also associated with a range of feeding practices, which are themselves linked to the quality of rangelands whose influence on the morphological development of animals has been widely observed.

Keywords: dromedary, female, body measurement, animal population, Algeria

\section{Resumen}

Babelhadj B., Benaissa A., Adamou A., Tekkouk-Zemmouchi F., Raache S., Babelhadj T., Guintard C. Enfoque morfozoométrico de las camellas (Camelus dromedarius L.) en las poblaciones argelinas Sahraoui y Targui

El objetivo del presente estudio fue comparar los trazos morfológicos y determinar el índice de flexibilidad de dos poblaciones de camellos argelinos, Sahraoui y Targui, basándose en medidas zoométricas. Estas medidas concernieron una muestra de camellas adultas - 30 Sahraoui y 30 Targui - de más de cinco años en el Sahara del Norte (región de Ouargla). Se tomaron cuatro medidas, luego se calcularon el índice de flexibilidad y el peso vivo. Se proponen ecuaciones de regresión linear para estimar el peso vivo de los animales y la altura a la cruz a partir de medidas correlacionadas. El peso vivo medio de los Sahraoui y Targui fue $399 \pm 74 \mathrm{~kg}$ y $428 \pm 82 \mathrm{~kg}$ respectivamente para una altura a la cruz de $1,75 \pm 0,08 \mathrm{~m}$ y $1,81 \pm 0,08 \mathrm{~m}$. Las cavidades sub esternales promedio midieron 0,96 $\pm 0,05 \mathrm{~m}$ y $0,97 \pm 0,08 \mathrm{~m}$, la profundidad torácica media $0,80 \pm 0,08$ y $0,84 \pm 0,07$ y los índices de flexibilidad sub esternal fueron de $1,20 \pm 0,13 \mathrm{~m}$ y $1,18 \pm 0,16 \mathrm{~m}$. El presente estudio muestra que la camella Targui es ligeramente más grácil que la Sahraoui. Estas poblaciones se asocian también con una variabilidad de prácticas alimenticias, que están en si unidas a la calidad de los pastizales, cuya influencia en el desarrollo morfológico de los animales ha sido ampliamente observada.

Palabras clave: dromedario, hembra, medición del cuerpo, población animal, Argelia 
